\title{
Multi-scale catalytic fast pyrolysis of Grindelia reveals opportunities for generating low oxygen content bio-oils from drought tolerant biomass
}

Phillip Cross ${ }^{\mathrm{a} \dagger}$, Kristiina Iisa ${ }^{\mathrm{b} \dagger}$, Anh To ${ }^{\mathrm{b}}$, Mark Nimlos ${ }^{\mathrm{b}}$, Daniel Carpenter ${ }^{\mathrm{b}}$, Jesse

A. Mayer ${ }^{\mathrm{d}}$, John C. Cushman ${ }^{\mathrm{d}}$, Bishnu Neupane ${ }^{\mathrm{e}}$, Glenn C. Miller ${ }^{\mathrm{e}}$, Sushil

Adhikari $^{\mathrm{a}^{*}}$ and Calvin Mukarakate ${ }^{\mathrm{b} *}$

Number of pages: $\quad 14$

Number of Figures: $\quad 4$

Number of Tables: $\quad 5$

${ }^{*}$ Corresponding Author. Correspondence should be addressed to calvin.mukarakate@nrel.gov (C. Mukarakate) and sza0016@auburn.edu (S. Adhikari).

$\uparrow \mathrm{PC}$ and $\mathrm{KI}$ contributed equally to the article. 


\section{Catalyst characterizations}

Total acid site density was determined using ammonia temperature-programmed desorption ( $\mathrm{NH}_{3}-\mathrm{TPD}$ ) on an Altamira AMI-390 Instrument with gas flow rates of $25 \mathrm{smL} / \mathrm{min}$. Catalyst samples (ca. $100 \mathrm{mg}$ ) were pretreated in flowing He at $2{ }^{\circ} \mathrm{C} / \mathrm{min}$ to $120^{\circ} \mathrm{C}$ for $2 \mathrm{~h}$ to remove adsorbed moisture. The samples were then saturated with flowing $10 \% \mathrm{NH}_{3} / \mathrm{He}\left(25 \mathrm{smL} / \mathrm{min}\right.$ ) for $30 \mathrm{~min}$. Excess and/or physisorbed $\mathrm{NH}_{3}$ was removed with flowing $\mathrm{He}$ at $120^{\circ} \mathrm{C}$ for $1 \mathrm{~h}$. TPD of $\mathrm{NH}_{3}$ was performed by heating the sample from 120 to $500{ }^{\circ} \mathrm{C}$ at $30{ }^{\circ} \mathrm{C} / \mathrm{min}$, holding at $500{ }^{\circ} \mathrm{C}$ for $1 \mathrm{~h}$. Desorbed $\mathrm{NH}_{3}$ was measured with a TCD, and calibration was performed after each experiment by introducing 10 pulses of $10 \% \mathrm{NH}_{3} / \mathrm{He}$ through a 5 $\mathrm{mL}$ sample loop into a stream of flowing He.

$N_{2}$ physisorption was used to measure textural properties of the catalysts. Before measurement, the catalysts were pretreated at $150{ }^{\circ} \mathrm{C}$ under He flow for $>12 \mathrm{~h}$ to remove adsorbed moisture. $\mathrm{N}_{2}$ physisorption was performed at 77K using Quadrasorb Station 2 instrument. Surface area was determined using the Brunauer-Emmett-Teller (BET) method at relative pressure $\left(\mathrm{P} / \mathrm{P}_{\mathrm{o}}\right)$ below 0.1 , and total pore volume was calculated at $\mathrm{P} / \mathrm{P}_{\mathrm{o}}$ of 0.995 .

Thermogravimetric analysis coupled with Fourier transform infrared (TGA-FTIR) spectroscopy was conducted using a SYS Evolution TGA instrument coupled with a Thermo Scientific Nicolet 6700 FTIR spectrophotometer to quantify coke deposit on the spent catalysts. In a typical analysis, ca. $100 \mathrm{mg}$ of spent catalyst was pretreated at $150^{\circ} \mathrm{C}$ for $1 \mathrm{~h}$ in $25 \mathrm{sccm}$ flowing $\mathrm{N}_{2}$ to remove adsorbed moisture. Then, the carrier gas was switched to air $(25 \mathrm{sccm})$ at $150^{\circ} \mathrm{C}$ and held for $10 \mathrm{~min}$ to stabilize the baseline signals for both TGA and IR spectroscopy. The sample was then heated to $800^{\circ} \mathrm{C}$ at $20^{\circ} \mathrm{C} / \mathrm{min}$ in flowing air.

\section{GC-MS Analysis of Liquids from the $\mathbf{2}$ Inch Fluidized Bed Reactor (2FBR) system}

GC-MS analysis of the liquid samples was performed using an Agilent G1530A GC equipped with an Agilent 5973 mass-selective detector. Oil samples were diluted 1:20 in acetone and aqueous phase samples 1:1 in methanol. $1 \mathrm{ml}$ samples were injected into the GC, which contained a $30 \mathrm{~m} \times 0.25 \mathrm{~mm} \times$ $0.25 \mu \mathrm{m}$ Restek Rtx-50 (50\%-phenyl-methylpolysiloxane phase) column. The GC oven temperature was held at $40^{\circ} \mathrm{C}$ for $2 \mathrm{~min}$, ramped to $140^{\circ} \mathrm{C}$ at $7^{\circ} \mathrm{C} / \mathrm{min}$, then to $290^{\circ} \mathrm{C}$ at $12^{\circ} \mathrm{C} / \mathrm{min}$ and held for $5 \mathrm{~min}$. The inlet temperature was $250^{\circ} \mathrm{C}$, transfer line temperature $300^{\circ} \mathrm{C}$, and there was a helium carrier gas flow of $1 \mathrm{ml} / \mathrm{min}$ with a split ratio of 10:1. Semi-quantitative analysis was performed based on a calibration standard including seventeen compounds typical for biomass CFP compounds. Response factors for compounds not included in the standard were based on compounds with similar structure and molecular weight. Unknowns were quantified based on an average response factor. The liquid standard contained a mixture of hydrocarbons and oxygenated compounds: acetaldehyde, benzene, 2,5dimethylfuran, toluene, ethylbenzene, $\mathrm{p}$-xylene, 2-furaldehyde, 2-cyclopentenone, phenol, 1,2,3trimethylbenzene, 2,3- benzofuran, indene, o-cresol, o-methoxyphenol, 1-naphthol, naphthalene, 2methylnaphthalene, phenanthrene, n-octane, 1-octene, cis-1,4-dimethyl-cyclohexane, n-decane, and tetralin. 


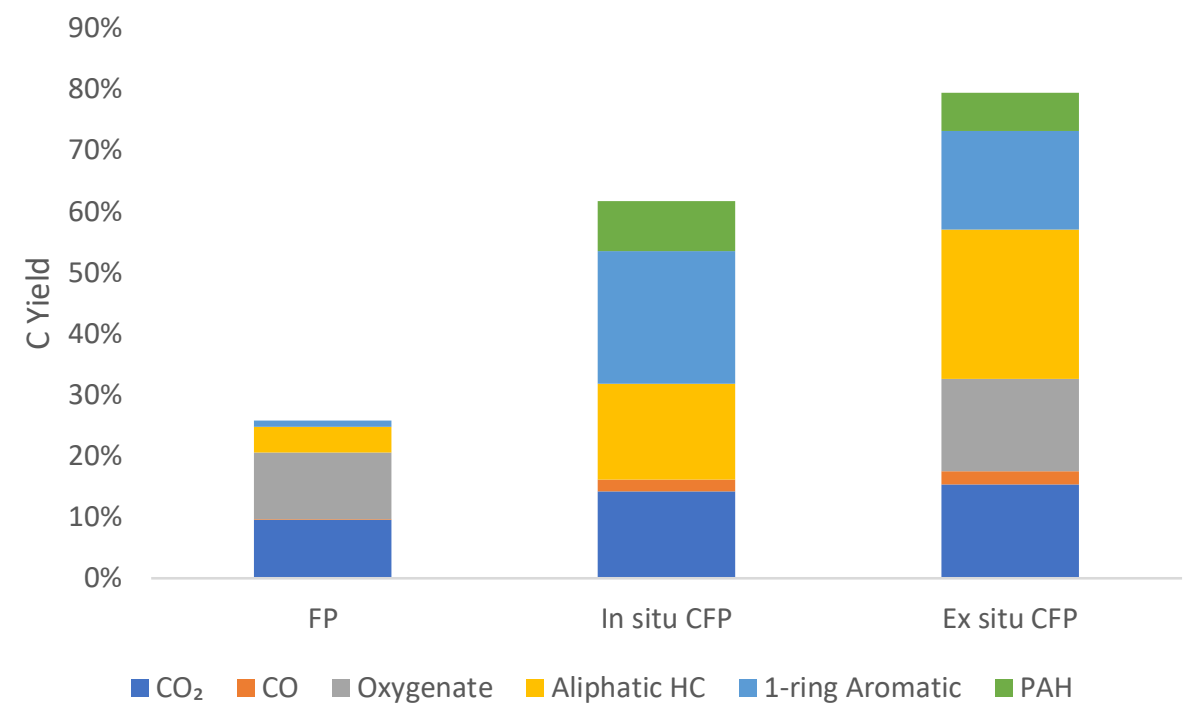

Figure S1. Distribution of gaseous and vapor products within major categories in the tandem micro pyrolyzer experiments. Biomass:Catalyst 0.1 in the catalytic experiments; temperature $550^{\circ} \mathrm{C}$. The unidentified compounds consist of solids (char and/or coke) and non-GCMS detectable vapors such as high-molecular weight compounds. 
Table 1. Tandem microreactor compounds for fast pyrolysis of Grindelia. The compound identities are based on matches to NIST database and have not been verified.

\begin{tabular}{|c|c|c|c|}
\hline $\begin{array}{l}\text { Time, } \\
\min \end{array}$ & Library/ID & $\begin{array}{l}\text { Avg. } \\
\text { wt\% }\end{array}$ & $\begin{array}{l}\text { St.dev } \\
\text { wt\%. }\end{array}$ \\
\hline 2.13 & Carbon dioxide & 14.4 & 8.3 \\
\hline 2.17 & Propene & 0.6 & 0.4 \\
\hline 2.31 & Acetaldehyde & 0.9 & 0.5 \\
\hline 2.37 & 1-Propene, 2-methyl- & 0.9 & 0.5 \\
\hline 2.73 & Acetone & 1.4 & 0.8 \\
\hline 2.81 & Furan & 0.5 & 0.3 \\
\hline 2.92 & 1,4-Pentadiene & 0.4 & 0.2 \\
\hline 3.03 & Acetic acid, methyl ester & 0.5 & 0.3 \\
\hline 3.17 & 1,3-Cyclopentadiene & 0.2 & 0.1 \\
\hline 3.64 & 2-Pentanone & 0.6 & 0.4 \\
\hline 3.78 & 2-Butanone & 0.5 & 0.3 \\
\hline 4.00 & Carbon Monoxide (TCD) & 0.2 & 0.2 \\
\hline 4.47 & Acetic acid & 1.2 & 0.7 \\
\hline 5.07 & 2-Propanone, 1-hydroxy- & 0.7 & 0.4 \\
\hline 6.48 & Toluene & 0.3 & 0.2 \\
\hline 7.81 & Butanoic acid, 3-methyl- & 0.5 & 0.3 \\
\hline 7.92 & Butanoic acid, 2-methyl- & 0.5 & 0.3 \\
\hline 8.06 & Cyclopentane, 2-ethylidene-1,1-dimethyl- & 0.4 & 0.3 \\
\hline 8.30 & 1,3,5,7-Cyclooctatetraene & 0.2 & 0.1 \\
\hline 8.56 & 1,2-Cyclopentanedione & 0.5 & 0.3 \\
\hline 9.13 & Phenol & 1.0 & 0.6 \\
\hline 9.66 & Limonene & 0.3 & 0.2 \\
\hline 10.04 & Phenol, 3-methyl- & 0.6 & 0.4 \\
\hline 11.71 & 2-Methoxy-4-vinylphenol & 0.6 & 0.4 \\
\hline 12.11 & Naphthalene, 1,2,3,4-tetrahydro-1,1,6-trimethyl- & 0.3 & 0.2 \\
\hline 12.60 & Phenol, 2-methoxy-4-(1-propenyl)- & 0.5 & 0.3 \\
\hline 13.18 & $\begin{array}{l}\text { 1H-Cycloprop[e]azulene, 1a,2,3,4,4a,5,6,7b-octahydro-1,1,4,7-tetramethyl-, } \\
\text { [1aR-(1a.alpha.,4.alpha.,4a.beta.,7b.alpha.)]- }\end{array}$ & 0.5 & 0.3 \\
\hline 15.41 & Benzene, 1-(5,5-dimethyl-1-cyclopenten-1-yl)-2-methoxy- & 0.9 & 0.5 \\
\hline 15.56 & Benzocyclopentene, 4,5,6,7-tetraethyl-1-methyl- & 0.5 & 0.3 \\
\hline
\end{tabular}


Table 2. Tandem microreactor compounds for in situ catalytic fast pyrolysis of Grindelia. The compound identities are based on matches to NIST database and have not been verified.

\begin{tabular}{|c|c|c|c|}
\hline RT, $\min$ & Library/ID & Average, wt\% & St.dev. \\
\hline 1.55 & Ethene & $5.8 \%$ & $1.6 \%$ \\
\hline 2.15 & Carbon dioxide & $14.3 \%$ & $2.0 \%$ \\
\hline 2.21 & Propene & $5.3 \%$ & $0.4 \%$ \\
\hline 2.34 & Isobutane & $0.9 \%$ & $0.2 \%$ \\
\hline 2.39 & 1-Propene, 2-methyl- & $0.9 \%$ & $0.0 \%$ \\
\hline 2.41 & Butane & $0.3 \%$ & $0.0 \%$ \\
\hline 2.46 & 1-Butene & $0.3 \%$ & $0.0 \%$ \\
\hline 2.50 & 1-Propene, 2-methyl- & $0.2 \%$ & $0.0 \%$ \\
\hline 2.72 & Butane, 2-methyl & $1.3 \%$ & $0.2 \%$ \\
\hline 4.02 & Carbon Monoxide (TCD) & $1.9 \%$ & $0.2 \%$ \\
\hline 5.15 & Benzene & $3.1 \%$ & $0.2 \%$ \\
\hline 6.87 & Toluene & $6.8 \%$ & $0.5 \%$ \\
\hline 8.02 & Ethylbenzene & $0.3 \%$ & $0.0 \%$ \\
\hline 8.12 & $\mathrm{p} / \mathrm{m}$-Xylene & $4.6 \%$ & $0.3 \%$ \\
\hline 8.36 & o-Xylene & $1.4 \%$ & $0.1 \%$ \\
\hline 9.03 & Benzene, 1-ethyl-2-methyl- & $0.3 \%$ & $0.0 \%$ \\
\hline 9.09 & Benzene, -trimethyl- & $0.3 \%$ & $0.0 \%$ \\
\hline 9.33 & Benzene, -trimethyl- & $1.2 \%$ & $0.0 \%$ \\
\hline 9.58 & Benzene, -trimethyl & $0.3 \%$ & $0.0 \%$ \\
\hline 9.70 & Indane & $0.3 \%$ & $0.0 \%$ \\
\hline 9.76 & Indene & $0.4 \%$ & $0.0 \%$ \\
\hline 10.11 & Benzene, 1-ethyl-2,3-dimethyl- & $0.3 \%$ & $0.0 \%$ \\
\hline 10.63 & 2-Methylindene & $0.3 \%$ & $0.0 \%$ \\
\hline 10.67 & 1H-Indene, 1-methyl- & $0.3 \%$ & $0.0 \%$ \\
\hline 10.90 & Naphthalene & $1.3 \%$ & $0.1 \%$ \\
\hline 10.99 & 1H-Indene, 2,3-dihydro-1,2-dimethyl- & $0.3 \%$ & $0.0 \%$ \\
\hline 11.38 & 1H-Indene, 2,3-dihydro-dimethyl- & $0.2 \%$ & $0.0 \%$ \\
\hline 11.49 & (1-Methylbuta-1,3-dienyl)benzene & $0.3 \%$ & $0.0 \%$ \\
\hline 11.62 & 1H-Indene, 2,3-dihydro-dimethyl- & $0.2 \%$ & $0.0 \%$ \\
\hline 11.70 & Naphthalene, -methyl- & $1.4 \%$ & $0.1 \%$ \\
\hline 11.82 & Naphthalene, -methyl- & $0.6 \%$ & $0.1 \%$ \\
\hline 11.87 & Naphthalene, -methyl & $0.3 \%$ & $0.1 \%$ \\
\hline 12.36 & Naphthalene, -dimethyl- & $0.4 \%$ & $0.0 \%$ \\
\hline 12.45 & Naphthalene, -dimethyl- & $0.6 \%$ & $0.0 \%$ \\
\hline 12.54 & Naphthalene, -dimethyl- & $0.4 \%$ & $0.0 \%$ \\
\hline 12.56 & Naphthalene, -dimethyl- & $0.5 \%$ & $0.0 \%$ \\
\hline 12.69 & Naphthalene, -dimethyl- & $0.4 \%$ & $0.0 \%$ \\
\hline 12.86 & Naphthalene, 1,2,3,4-tetrahydro-5,6,7,8-tetramethyl- & $0.1 \%$ & $0.1 \%$ \\
\hline 13.08 & Naphthalene, -trimethyl & $0.2 \%$ & $0.0 \%$ \\
\hline 13.15 & Naphthalene, 1,2,3,4-tetrahydro-5,6,7,8-tetramethyl- & $0.3 \%$ & $0.0 \%$ \\
\hline 13.20 & Naphthalene, -trimethyl & $0.3 \%$ & $0.0 \%$ \\
\hline
\end{tabular}


13.24 Naphthalene, -trimethyl

$0.3 \% \quad 0.0 \%$

13.33 Naphthalene, -trimethyl

$0.2 \% \quad 0.0 \%$

13.36 Naphthalene, -trimethyl

$0.2 \% \quad 0.0 \%$

13.45 Naphthalene, -trimethyl

$0.3 \% \quad 0.0 \%$

14.05 Naphthalene, 1,2,3,4-tetramethyl-

$0.1 \% \quad 0.0 \%$

14.14 Naphthalene, 1,2,3,4-tetramethyl-

$0.2 \% \quad 0.0 \%$

14.17 Azulene, 7-ethyl-1,4-dimethyl-

$0.1 \% \quad 0.1 \%$

14.27 Azulene, 7-ethyl-1,4-dimethyl-

$0.2 \% \quad 0.0 \%$

14.74 Anthracene

$0.3 \% \quad 0.0 \%$

14.90 Naphthalene, 1,4-dimethyl-7-(1-methylethyl)-

$0.2 \% \quad 0.0 \%$

15.38 Phenanthrene, 1-methyl-

$0.5 \% \quad 0.1 \%$ 
Table 3. Tandem microreactor compounds for ex situ catalytic fast pyrolysis of Grindelia for biomass:catalyst 0.1-2 g/g. The compound identities are based on matches to NIST database and have not been verified. Included are compounds present at $\geq 0.5 \mathrm{wt} \%$ by semiquantitative analysis; however, the categories in Fig. 3 of the manuscript are based on all measured peaks.

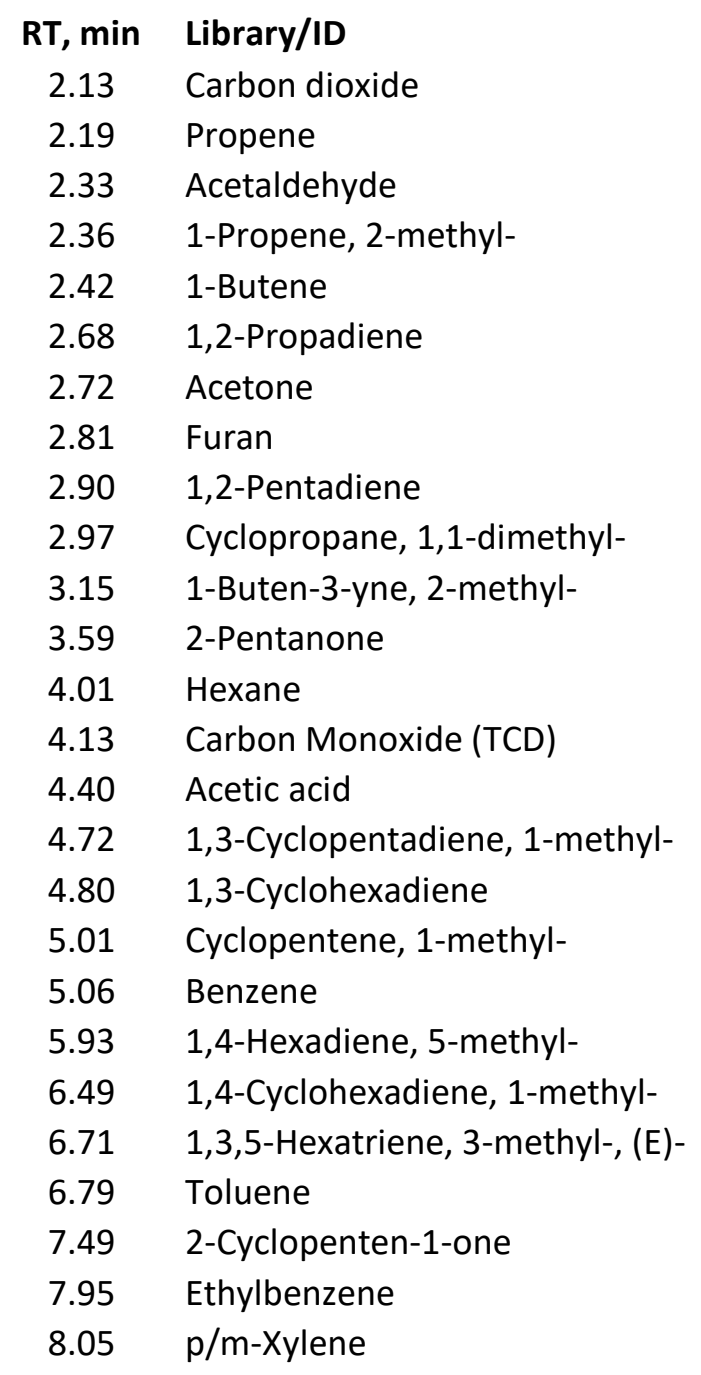

\begin{tabular}{ccccccccccc}
\multicolumn{10}{c}{ Biomass:Catalyst, g/g } \\
$\mathbf{0 . 1}$ & $\mathbf{0 . 2}$ & $\mathbf{0 . 4}$ & $\mathbf{0 . 6}$ & $\mathbf{0 . 8}$ & $\mathbf{1 . 0}$ & $\mathbf{1 . 2}$ & $\mathbf{1 . 4}$ & $\mathbf{1 . 6}$ & $\mathbf{1 . 8}$ & $\mathbf{2 . 0}$ \\
$\mathbf{2 3 . 2}$ & 25.6 & 19.9 & 20.2 & 21.0 & 20.8 & 21.1 & 19.6 & 20.5 & 20.7 & 18.7 \\
12.6 & 7.1 & 6.2 & 5.2 & 4.3 & 4.1 & 3.4 & 2.8 & 2.6 & 2.4 & 3.1 \\
& & 0.6 & 0.6 & 0.7 & 0.7 & 0.7 & 0.6 & 0.7 & 0.6 & 0.6 \\
9.3 & 6.1 & 6.4 & 6.1 & 5.5 & 5.6 & 5.5 & 3.8 & 3.7 & 4.8 & 4.0 \\
2.3 & 1.5 & 1.5 & 1.4 & 1.2 & 1.2 & 1.3 & 1.0 & 1.0 & 1.0 & 1.1 \\
1.7 & 1.1 & 0.9 & 0.9 & 1.0 & 0.9 & & 0.7 & 0.8 & 0.8 & \\
1.5 & 1.3 & 1.3 & 1.3 & 1.3 & 1.2 & 1.5 & 1.1 & 1.2 & 1.0 & 1.2 \\
1.4 & 1.1 & 1.2 & 1.2 & 1.2 & 1.1 & 1.2 & 1.1 & 1.0 & 1.0 & 1.1 \\
1.6 & 1.1 & 1.5 & 1.5 & 1.5 & 1.5 & 1.6 & 1.4 & 1.4 & 1.5 & 1.6 \\
2.6 & 2.1 & 2.1 & 1.9 & 1.7 & 1.8 & 1.7 & 1.4 & 1.5 & 1.4 & 1.5 \\
1.3 & 0.6 & 0.7 & 0.7 & 0.6 & 0.6 & 0.6 & 0.6 & 0.5 & 0.5 & 0.6 \\
& & & & 0.5 & 0.5 & 0.5 & 0.6 & 0.6 & 0.6 & 0.6 \\
0.8 & & 0.7 & & 0.5 & & 0.6 & 0.5 & & & 0.8 \\
1.1 & 1.5 & 1.1 & 1.0 & 1.1 & 0.8 & 1.0 & 1.0 & 1.0 & 0.9 & 0.9 \\
& & & 0.5 & 0.6 & 0.6 & 0.8 & 0.9 & 0.7 & 0.7 & 0.6 \\
0.9 & 0.6 & 0.7 & 0.7 & 0.7 & 0.7 & 0.7 & 0.6 & 0.6 & 0.5 & 0.7 \\
0.9 & 0.6 & 0.7 & 0.7 & 0.6 & 0.6 & 0.7 & 0.5 & 0.5 & 0.5 & 0.6 \\
0.7 & & & & & & & & & & \\
1.2 & 0.7 & 0.6 & 0.5 & 0.5 & & 0.5 & & & & 0.5 \\
0.5 & 0.5 & 0.7 & 0.6 & 0.7 & 0.7 & 0.8 & & & & 0.7 \\
0.6 & & & & & & & & & & 0.5 \\
0.5 & & & & & & & & & & \\
3.1 & 1.6 & 1.4 & 1.1 & 0.9 & 0.8 & 0.8 & 0.6 & 0.6 & 0.6 & 0.7 \\
& & & & & & & & & & 0.5 \\
0.7 & & & & & & & & & & \\
3.2 & 2.0 & 1.7 & 1.3 & 1.1 & 0.9 & 0.9 & 0.5 & 0.5 & & 0.6
\end{tabular}


8.29 o-Xylene

8.99

9.27

9.33

10.56

11.66

12.05

13.08

13.12

13.23

14.90

14.94

15.20

15.30

15.84

16.85
Benzene, 1-ethyl-4-methyl-

Benzene, 1,2,3-trimethyl-

1,5-Heptadien-3-yne

1H-Indene, 1-methyl-

Naphthalene, 1-methyl-

Naphthalene, 1,2,3,4-tetrahydro-1,1,6-trimethyl-

1H-Inden-1-one, 2,3-dihydro-3,3,4,6-tetramethyl-

1H-3a,7-Methanoazulene, octahydro-1,9,9-trimethyl-4methylene-, (1.alpha.,3a.alpha.,7.alpha.,8a.beta.)-

Naphthalene, 1,2-dihydro-2,5,8-trimethyl-

5-[2-(5-Oxopenta-1,3-dienyl)phenyl]penta-2,4-dienal 2(1H)-Isoquinolinecarboxylic acid, 1-cyano-1-methyl-, ethyl ester

Bicyclo[3.1.0]hexan-2-one, 6,6-dicyano-5-(1cyclohepten-1-yl)-

Naphthalene, 2-methyl-1-nitro-

2-Naphthaleneacetic acid, 6-methoxy-.alpha.-methyl-, methyl ester

Hexadecane $\begin{array}{llll}0.8 & 0.5 & 0.5 & 0.5\end{array}$

$\begin{array}{lll}0.7-0.6 & 0.5\end{array}$

$\begin{array}{llll}1.3 & 0.6 & 0.8 & 0.6\end{array}$

$\begin{array}{lll}0.8 & 0.8 & 0.7\end{array}$

$\begin{array}{lll}0.8 & 0.5 & 0.5\end{array}$

$0.5 \quad 0.5$

$\begin{array}{lllllll}0.5 & 0.6 & 0.6 & 0.6 & 0.6 & 0.6 & 0.6\end{array}$

$\begin{array}{lllllll}0.5 & 0.5 & 0.5 & 0.6 & 0.5 & 0.5 & 0.5\end{array}$

0.5

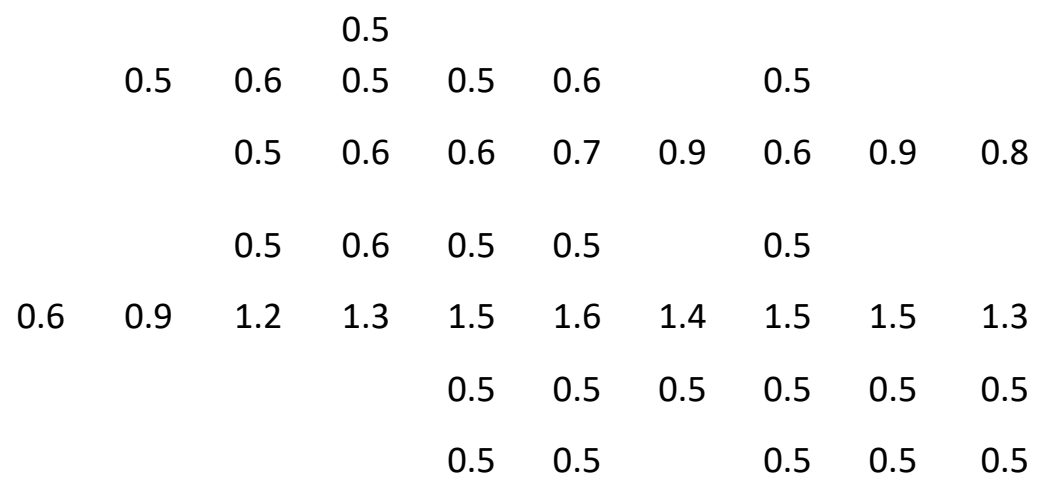




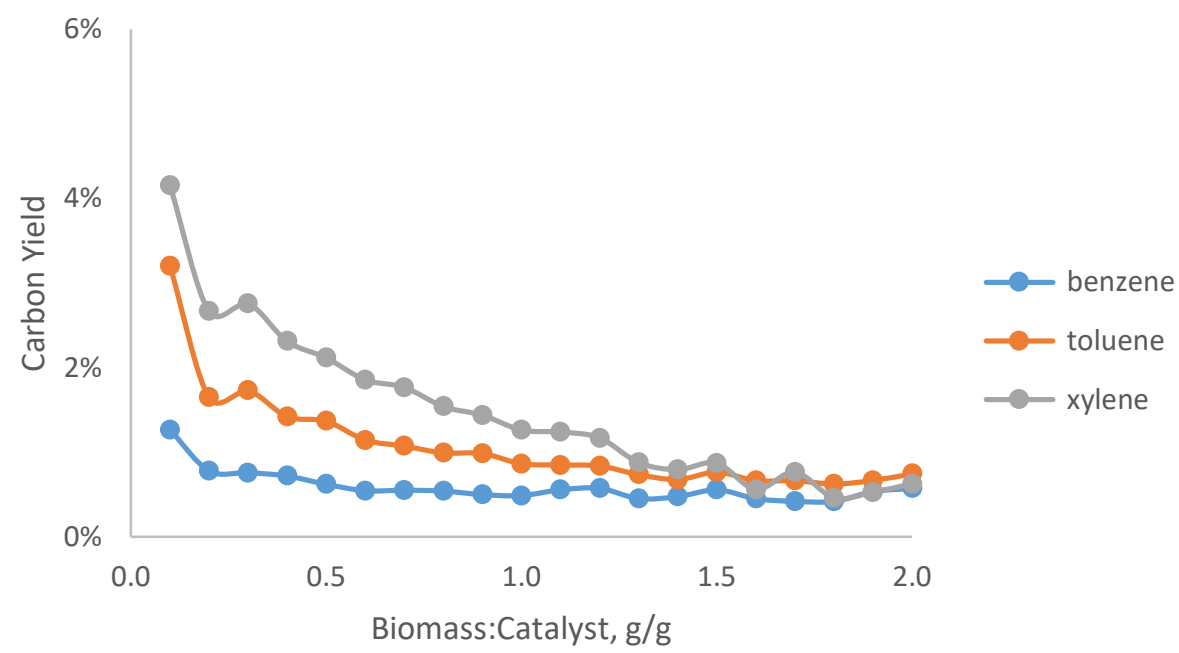

a)

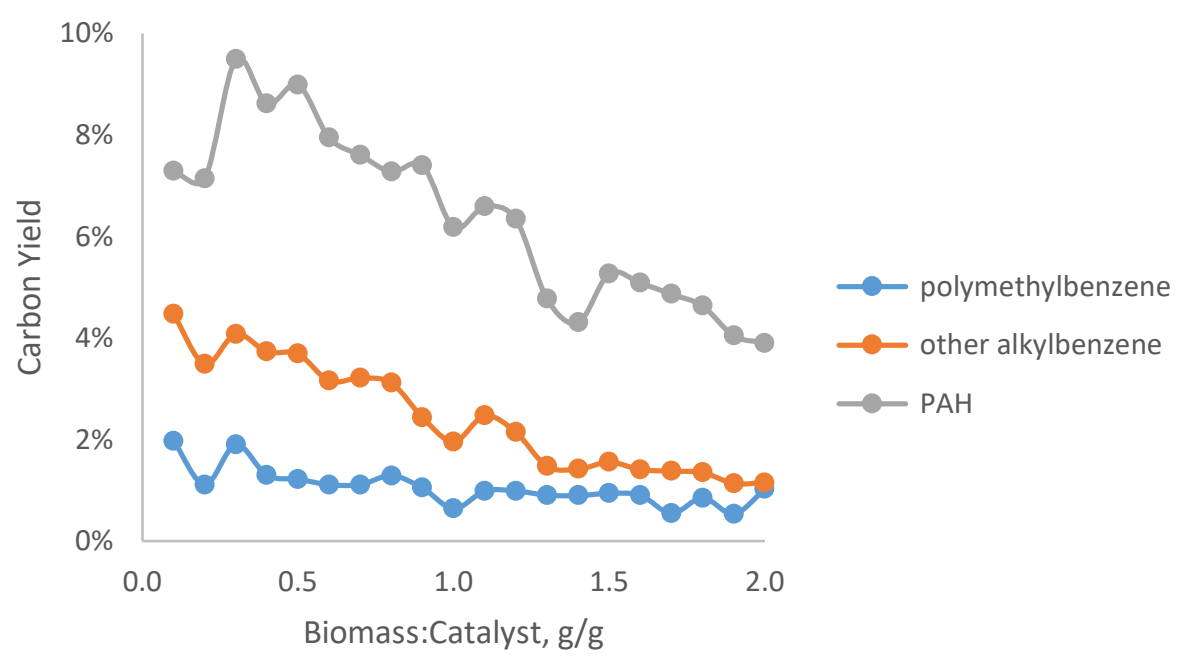

b)

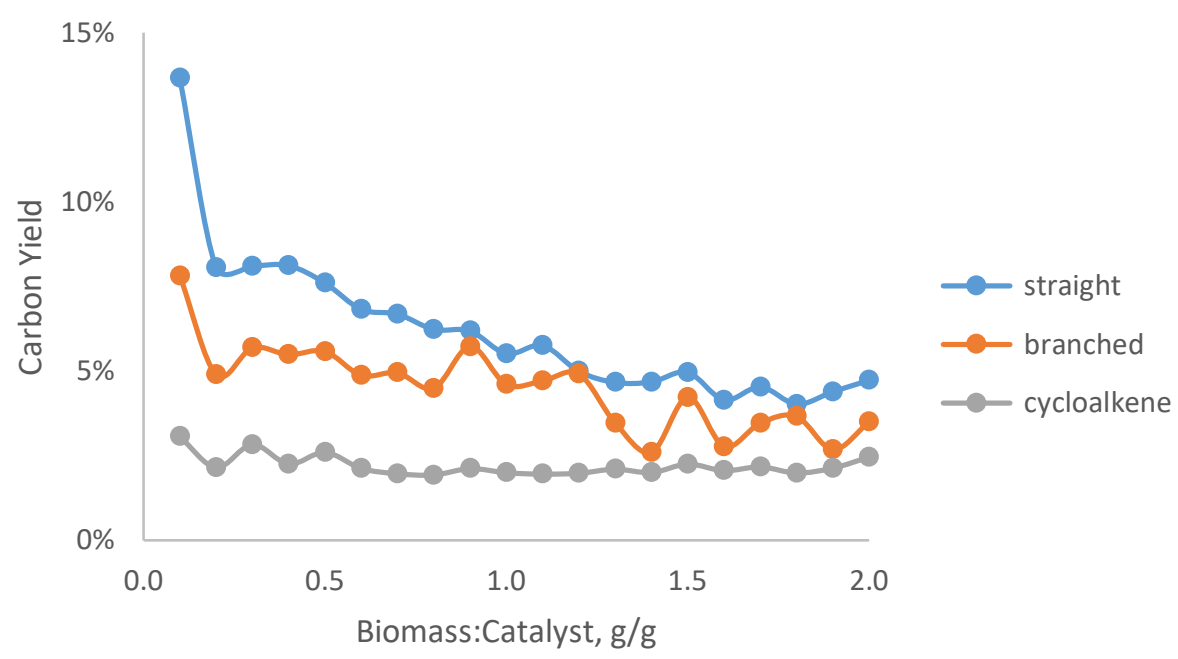

c) 


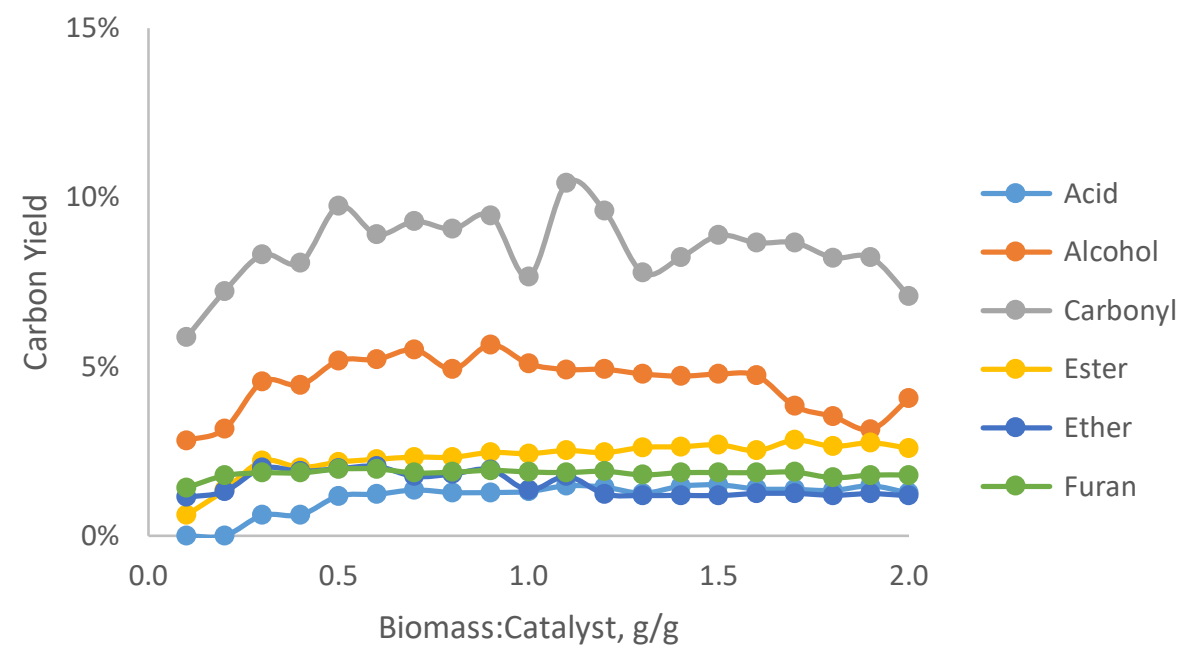

d)

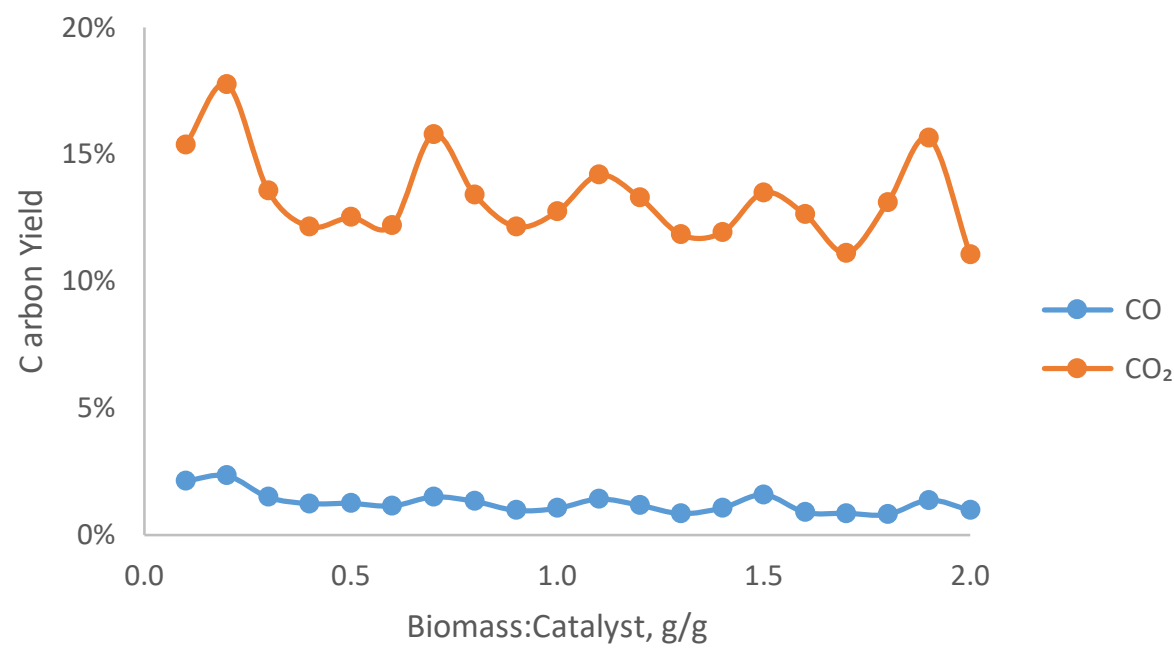

e)

Figure S2. Carbon yields for a) benzene, toluene, xylene, b) other aromatic hydrocarbons, c) alkenes, d) oxygenates, and e) CO and $\mathrm{CO}_{2}$ during ex situ CFP in the tandem micro pyrolyzer up to biomass:catalyst of 2 . 

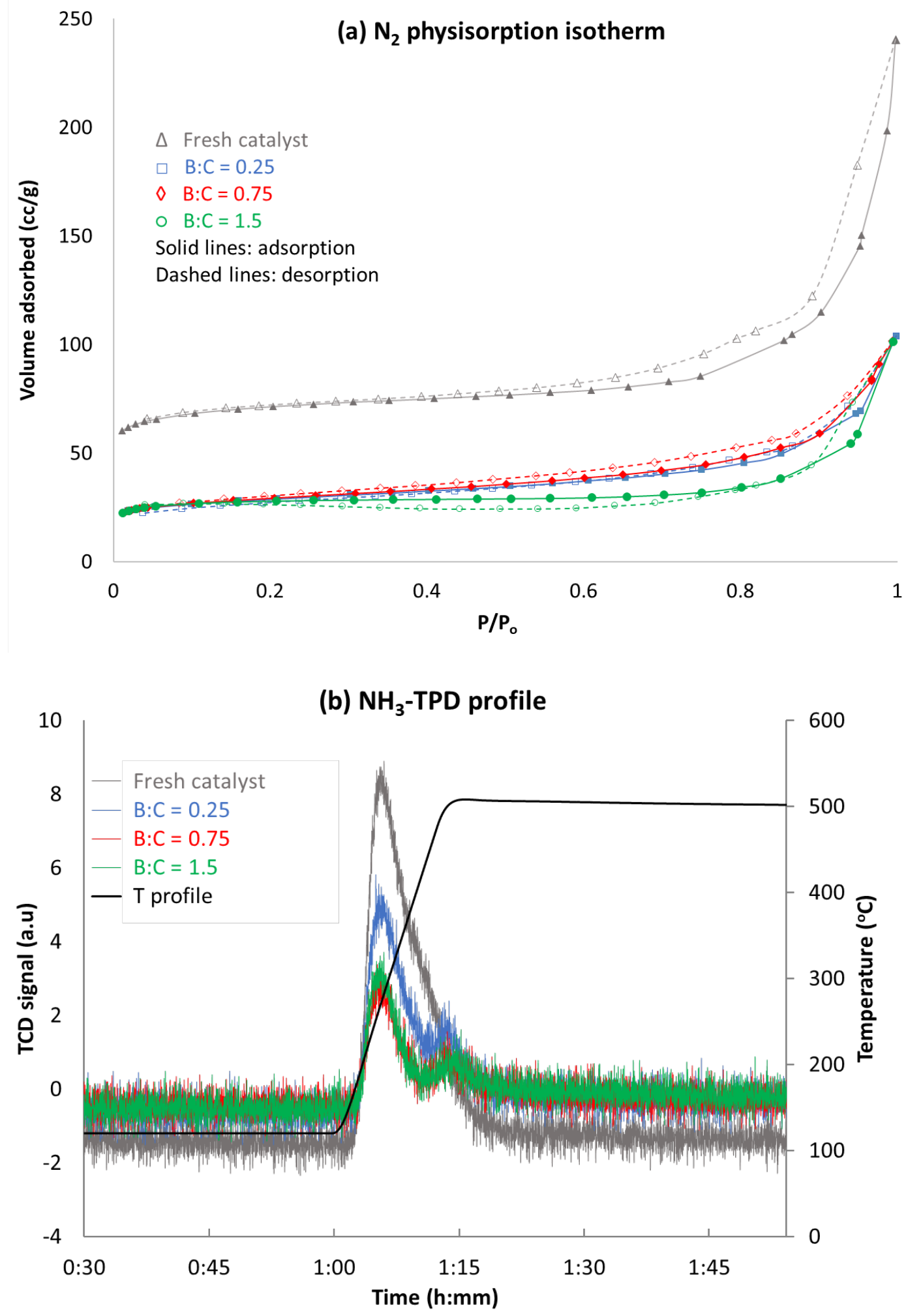

Figure S3. a) $\mathrm{N}_{2}$ physisorption isotherm and b) $\mathrm{NH}_{3}$ desorption profile for fresh and post-reaction catalysts from Py-MBMS experiments with different $\mathrm{B}: \mathrm{C}$ ratios of $0.5,1.5$ and 3.0. 


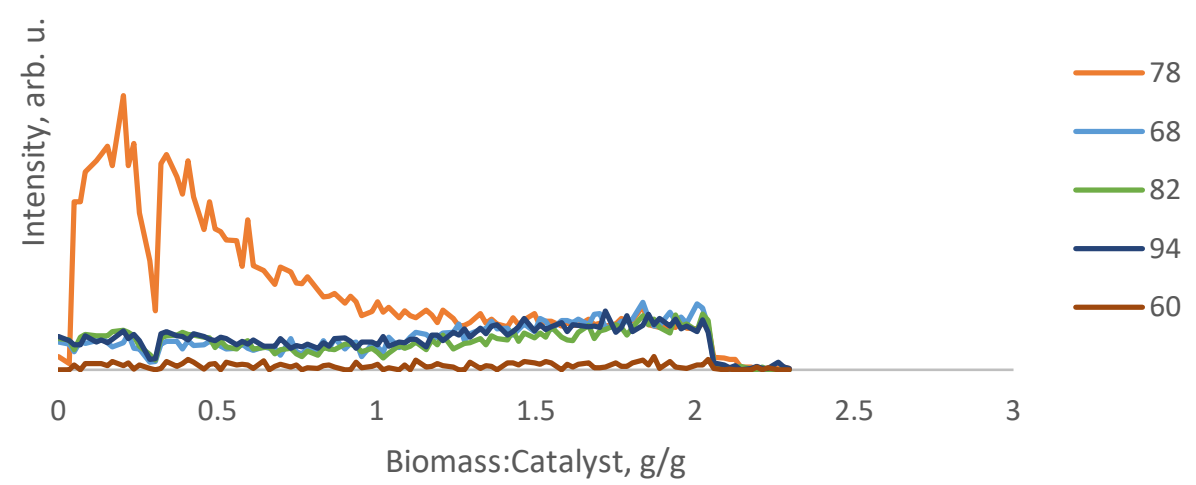

a)

\section{Ex situ CFP of Gumweed}

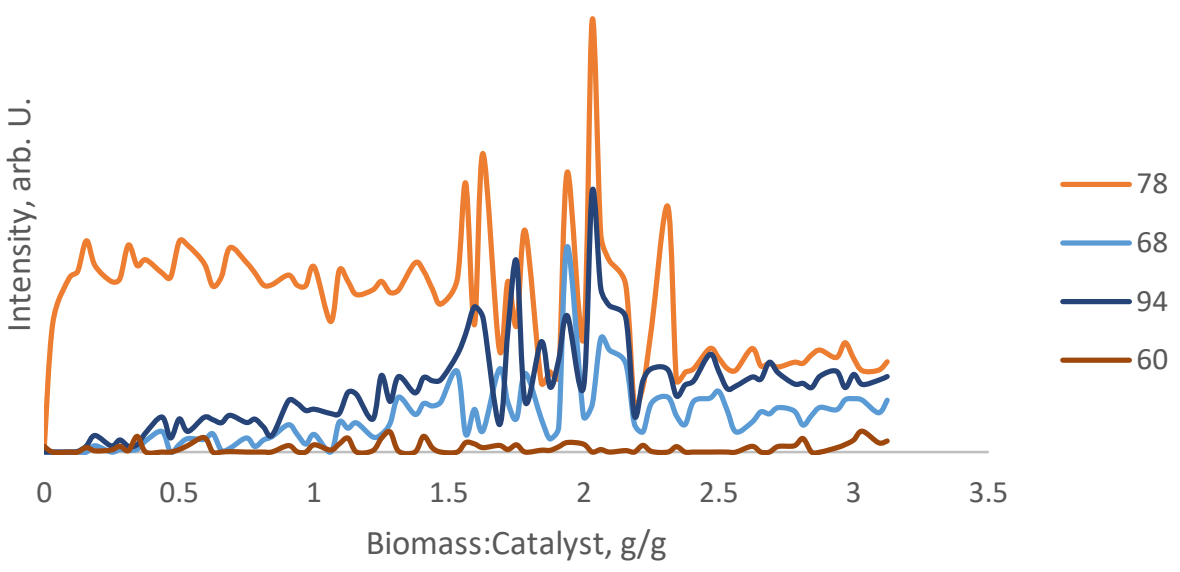

b)

Figure S4. Residual gas analyzer (RGA) traces for selected $\mathrm{m} / \mathrm{z}$ values for a) in situ and b) ex situ CFP of gumweed in the benchscale experiment. Hydrocarbon signals ( $\mathrm{m} / \mathrm{z} 78$ for benzene) decay more slowly for the ex situ experiment, suggesting slower deactivation than for the in situ experiment. The signals for partially upgraded compounds ( $\mathrm{m} / \mathrm{z} 68$ and 94 for furan and phenol, respectively) slightly increase and the signal for primary vapors ( $\mathrm{m} / \mathrm{z} 60$ for acetic acid or hydroxyacetaldehyde) are very low in both cases. The intensities are in arbitrary units. The noisy signals towards the end of the ex situ experiment may be due to blockage of the capillary leading to the RGA. 
Table S4. Metal contents in feedstock and char for bench-scale experiments in the 2-inch fluidized bed reactor system . Cd, Co, $\mathrm{Cr}, \mathrm{Cu}, \mathrm{Ni}$, and $\mathrm{Pb}$ were below the detection limit (500 ppm) in all samples.

\begin{tabular}{lcccccccccc} 
Ppm (wt) & Al & Ca & Cu & Fe & K & Mg & Mn & Na & P & S \\
\hline Grindelia feed & 3,153 & 13,257 & $<500$ & 3,303 & 28,788 & 2,855 & $<500$ & 2,047 & 2,123 & 2,894 \\
FP char & 4,390 & 28,878 & $<500$ & 3,747 & 54,954 & 6,274 & 693 & 2,046 & 4,550 & 3,196 \\
In situ CFP char & 4,677 & 49,044 & 514 & 4,127 & 94,301 & 11,249 & 604 & 2,307 & 7,893 & 5,430 \\
Ex situ CFP char & 5,144 & 46,600 & $<500$ & 4,858 & 92,194 & 9,230 & $<500$ & 2,084 & 7,699 & 5,067 \\
\hline Remaining in char & & & & & & & & & & \\
FP char & $33 \%$ & $52 \%$ & n/a & $27 \%$ & $46 \%$ & $53 \%$ & n/a & $24 \%$ & $51 \%$ & $27 \%$ \\
In situ CFP char & $34 \%$ & $85 \%$ & n/a & $29 \%$ & $76 \%$ & $91 \%$ & n/a & $26 \%$ & $86 \%$ & $43 \%$ \\
Ex situ CFP char & $23 \%$ & $49 \%$ & n/a & $20 \%$ & $45 \%$ & $45 \%$ & n/a & $14 \%$ & $50 \%$ & $24 \%$ \\
\hline
\end{tabular}

Table S5. Concentrations in wt\% in liquid for main compounds in the liquid phases. Included are compounds present at $\geq 0.5$ wt\% by semiquantitative analysis; however, the categories in Fig. 3 are based on all measured peaks. Compounds with matches $<70 \%$ are labelled as unknown. The identities are based on matches to NIST library and have not been verified.

RT,

min Compound

3.11 Acetic acid

$6.22 \mathrm{p} / \mathrm{m}$-Xylene

10.45 Phenol

11.99 Phenol, 2-methyl-

12.14 2-Cyclopenten-1-one, 2-hydroxy-3-methyl-

12.55 Phenol, 4-methyl-

13.62 2-Methylindene

14.00 Phenol, 2,4-dimethyl-

14.51 Phenol, 2-ethyl-

15.04 Naphthalene

17.02 Quinoline

17.11 Naphthalene, 2-methyl-

17.80 2-Methoxy-4-vinylphenol

17.90 Unknown

17.94 Naphthalene, 1,2,3,4-tetrahydro-1,5,8-trimethyl-

$18.121 \mathrm{H}$-Inden-1-one, 2,3-dihydro-

18.13 Hydroquinone $=$ Benzene-1,4-diol

18.34 Indole

18.42 Unknown

18.74 Naphthalene, 2,6-dimethyl-

18.81 Quinoline, 5-methyl-

18.91 Quinoline, 3-methyl-

$18.921 \mathrm{H}$-Indenol

1H-Cycloprop[e]azulene, 1a,2,3,4,4a,5,6,7b-octahydro-1,1,4,7-

19.11 tetramethyl-, [1aR-(1a.alpha.,4.alpha.,4a.beta.,7b.alpha.)]-

$19.551 \mathrm{H}-$ Indole, 3-methyl-
In Situ Ex situ

FP Oil FP aq ZSM5 ZSM5

$0.7 \%$

$0.6 \%$

$0.6 \% \quad 1.6 \% \quad 2.5 \%$

$0.7 \% \quad 1.1 \%$

$1.3 \%$

$1.4 \% \quad 1.9 \%$

$0.6 \%$

$0.6 \% \quad 1.1 \%$

$0.7 \%$

$0.5 \%$

$0.5 \%$

$0.9 \% \quad 1.3 \%$

$0.6 \%$

$0.5 \%$

$0.6 \% \quad 0.6 \%$

$0.5 \%$

$1.3 \% \quad 1.2 \%$

$0.8 \%$

$0.5 \% \quad 0.7 \%$

$0.5 \%$

$0.6 \%$

$0.7 \%$

$0.9 \%$

$0.6 \% \quad 1.1 \%$ 
$19.641 \mathrm{H}$-Indole, 4-methyl-

19.75 Eugenol = Phenol, 2-methoxy-4-(1-propenyl)-

$0.5 \%$

$0.7 \%$

19.83 1H-Inden-1-one, 2,3-dihydro-3,3,5,6-tetramethyl-

$3.9 \% \quad 5.4 \%$

20.14 Unknown

$0.6 \% \quad 0.7 \%$

20.65 Naphthalene, 1,6,7-trimethyl-

$0.5 \%$

20.75 Indolizine, 2,7-dimethyl-

$0.6 \%$

20.87 Unknown

$1.4 \%$

20.92 (3.alpha.,3a.beta.,6.alpha.,7.beta.,8a.alpha.)]-

$0.8 \%$

$21.201 \mathrm{H}$-Indene, 5-(1,1-dimethylethyl)-2,3-dihydro-1,1-dimethyl-

$0.8 \%$

21.52 Unknown

21.73 Unknown

$0.5 \%$

22.21 Fluorene

$0.6 \%$

22.27 Unknown

$0.7 \%$

$0.5 \%$

22.39 1-Naphthalenol, 4-methyl-

$0.7 \%$

$0.6 \% \quad 0.7 \%$

22.90 Hexadecanoic acid, methyl ester

$0.5 \%$

23.45 1-Naphthol, 5,7-dimethyl-

$0.5 \%$

23.57 Unknown

$1.2 \%$

23.95 Unknown

$0.9 \%$

$0.8 \%$

$1.0 \%$

24.04 Unknown

$1.2 \%$

24.41 Hexamethyl-2,3-dihydro-benzofuran

$0.6 \%$

24.63 Unknown

$0.5 \%$

24.76 Unknown

$0.6 \%$

24.93 Unknown

$0.6 \%$ 